\title{
REEB DYNAMICS INSPIRED BY KATOK'S EXAMPLE IN FINSLER GEOMETRY
}

\author{
PETER ALBERS, HANSJÖRG GEIGES, AND KAI ZEHMISCH
}

\begin{abstract}
Inspired by Katok's examples of Finsler metrics with a small number of closed geodesics, we present two results on Reeb flows with finitely many periodic orbits. The first result is concerned with a contact-geometric description of magnetic flows on the 2-sphere found recently by Benedetti. We give a simple interpretation of that work in terms of a quaternionic symmetry. In the second part, we use Hamiltonian circle actions on symplectic manifolds to produce compact, connected contact manifolds in dimension at least five with arbitrarily large numbers of periodic Reeb orbits. This contrasts sharply with recent work by Cristofaro-Gardiner, Hutchings and Pomerleano on Reeb flows in dimension three. With the help of Hamiltonian plugs and a surgery construction due to Laudenbach we reprove a result of Cieliebak: one can produce Hamiltonian flows in dimension at least five with any number of periodic orbits; in dimension three, with any number greater than one.
\end{abstract}

\section{INTRODUCTION}

In a much-cited paper, Katok [19] constructed non-reversible Finsler metrics on spheres and projective spaces with small numbers of closed geodesics (and an ergodic geodesic flow) by deforming a Riemannian metric all of whose geodesics are closed. The geometry of these examples has been analysed in great detail by Ziller [25]. For the Katok metrics on the 2-sphere, a contact-geometric interpretation of the geodesic flow on the unit tangent bundle (or its double cover, the 3-sphere) as a Reeb flow was given by Harris and Paternain [17.

Geodesic (or rather cogeodesic) flows can be interpreted as Hamiltonian flows with respect to the canonical symplectic form on the cotangent bundle and the Hamiltonian function given by the square of the fibre norm. The Katok deformation of the Riemannian metric into a Finsler metric translates into a deformation of the Hamiltonian function.

Magnetic flows are generalisations of these geodesic flows; here the canonical symplectic form is modified by adding a closed 2-form lifted from the base of the cotangent bundle. Benedetti [2] recently extended the work of Harris-Paternain and gave an interpretation of magnetic flows on the 2-sphere (including their deformations into flows with only two periodic orbits) in terms of Reeb flows.

Katok takes a Riemannian manifold whose geodesic flow is periodic and alters the flow with the help of an $S^{1}$-action on that manifold. For instance, one starts with the geodesic flow of the round metric on the 2 -sphere $S^{2}$, and modifies it with the help of the rotation of $S^{2}$ about a fixed axis into a non-reversible Finsler metric

2010 Mathematics Subject Classification. 37J45; 37J55, 53D25, 53D35.

This research is part of a project in the SFB/TRR 191 'Symplectic Structures in Geometry, Algebra and Dynamics', funded by the DFG. 
of Randers type. Only the equator (traversed in either direction) survives as a closed geodesic.

In the present paper we discuss two constructions inspired by these examples. In Section 2 we show that Benedettis's work admits a strikingly simple interpretation in terms of a quaternionic symmetry of the 3-sphere. It is well known that the standard Reeb flow on the 3-sphere, which defines the Hopf fibration, can be perturbed into a Reeb flow with only two periodic orbits, seen for instance as the Reeb flow on an irrational ellipsoid in $\mathbb{R}^{4}$. We show that Benedetti's magnetic flows are nothing but quaternionic rotates of this example. The detailed formulation of the result is given in Theorem 2.3. Here is a précis.

Magnetic flows are Hamiltonian flows in the cotangent bundle of a manifold with respect to a symplectic form obtained by adding a closed 2 -form lifted from the base to the canonical symplectic form. As Hamiltonian function we take the fibre norm squared, and we study the dynamics on the unit cotangent bundle. When the base manifold is the 2-sphere $S^{2}$, it is convenient to pass to the double cover $S^{3}$ of the unit cotangent bundle.

Now, on $S^{3}$ there is a natural $S^{2}$-family of contact forms induced from a 2 -sphere of complex structures on $\mathbb{R}^{4}$, regarded as the space of quaternions. Writing $\mathbf{i}, \mathbf{j}, \mathbf{k}$ for the standard quaternionic units and $r$ for the radial coordinate on $\mathbb{R}^{4}$, we set $\alpha_{\mathbf{i}}=-\left.2 \mathrm{~d} r \circ \mathbf{i}\right|_{T S^{3}}$; the contact forms $\alpha_{\mathbf{j}}$ and $\alpha_{\mathbf{k}}$ are defined analogously. The triple $\left(\alpha_{\mathbf{i}}, \alpha_{\mathbf{j}}, \alpha_{\mathbf{k}}\right)$ is a taut contact sphere in the sense of [1].

We show that magnetic flows on (the unit cotangent bundle of) $S^{2}$, for the magnetic term being a multiple of the standard area form, are simply the Reeb flows in the circular family $\alpha^{\theta}:=\cos \theta \alpha_{\mathbf{i}}+\sin \theta \alpha_{\mathbf{j}}$ of contact forms. All these Reeb flows are periodic. The Reeb flow of $\alpha_{\mathbf{i}}$ corresponds to an infinite magnetic term and defines the Hopf fibration, as the double cover of the unit cotangent bundle of $S^{2}$. The Reeb flow of $\alpha_{\mathbf{j}}$ corresponds to a zero magnetic term and constitutes the geodesic flow. The deformation of the Hopf fibration into a flow with only two periodic orbits is then effected simultaneously on the whole circular family of Reeb flows.

The second part of the paper is concerned with the construction of Reeb and Hamiltonian flows with large but finite numbers of periodic orbits. In Section 3 we build on the idea of modifying a given Reeb flow on a bundle with the help of an $S^{1}$-action on the base manifold. Specifically, we start with the Reeb flow along the fibres of an $S^{1}$-bundle over a symplectic manifold coming from the classical Boothby-Wang construction [3], cf. [9, Section 7.2]. We then modify this periodic flow with the help of a Hamiltonian $S^{1}$-action on the base. In dimension five, for instance, this allows one to realise any integer $\geq 3$ as the number of periodic Reeb orbits on some closed, connected contact manifold. This contrasts sharply with recent results of Cristofaro-Gardiner, Hutchings and Pomerleano [7. In essence they established that, in dimension three, a contact manifold carries either two or infinitely many periodic Reeb orbits.

Using in addition a surgery construction for Hamiltonian flows due to Laudenbach [20] and Hamiltonian plugs [14, we can give a new proof of a result by Cieliebak [5]: Any non-negative integer (in dimension three: any integer $\geq 2$ ) can be realised as the number of closed characteristics on some hypersurface in the standard symplectic $\mathbb{R}^{2 n+2}$, i.e. as periodic orbits of a Hamiltonian flow (Theorem 3.2). 
Sections 2 and 3 are to a large part independent of each other.

\section{A QUATERnionic VieW OF MAGNetic Flows ON THE 2-SPhere}

On the cotangent bundle $T^{*} S^{2}$ of the 2-sphere $S^{2}$ we consider the canonical Liouville form $\lambda$, defined by $\lambda_{\mathbf{v}^{*}}=\mathbf{v}^{*} \circ T \pi$ at $\mathbf{v}^{*} \in T^{*} S^{2}$, where $\pi: T^{*} S^{2} \rightarrow S^{2}$ denotes the bundle projection. Given any (closed) 2-form $\sigma$ on $S^{2}$, which is referred to as the magnetic field, we can define a symplectic form

$$
\omega_{\sigma}:=\mathrm{d} \lambda-\pi^{*} \sigma
$$

on $T^{*} S^{2}$. The Hamiltonian vector field $X_{H}$ corresponding to a smooth function $H: T^{*} S^{2} \rightarrow \mathbb{R}$ is then defined by

$$
\omega_{\sigma}\left(X_{H}, .\right)=-\mathrm{d} H \text {. }
$$

If $g$ is a Riemannian metric on $S^{2}$ and \|\|$_{*}$ the dual norm on $T^{*} S^{2}$, the flow of the Hamiltonian vector field defined by the Hamiltonian function $H\left(\mathbf{v}^{*}\right)=\frac{1}{2}\left\|\mathbf{v}^{*}\right\|_{*}^{2}$ is called the magnetic flow of the pair $(g, \sigma)$.

Remark 2.1. The Hamiltonian flow for the symplectic form $\mathrm{d} \lambda$ is precisely the (co-)geodesic flow of $g$. In [9, Theorem 1.5.2] this is verified for the unit cotangent bundle $H^{-1}(1 / 2)$ of any Riemannian manifold. Since $H$ scales quadratically in the fibres of the cotangent bundle, the Hamiltonian vector field scales linearly in the fibres, as it should.

Now let $\sigma_{0}$ be the standard symplectic form on $S^{2}$ of total area $4 \pi$, corresponding to the round metric $g_{0}$ on $S^{2}$ of constant Gauß curvature 1 . We want to study the magnetic flow of the pairs $\left(g_{0}, s \sigma_{0}\right)$ for $s \in[0, \infty)$. For $s=0$ we have the geodesic flow; the projected flow lines on $S^{2}$ are the great circles. As the strength of the magnetic field increases, the projected flow lines on $S^{2}$ acquire an increasing left drift, causing the great circles for $s=0$ to become circles on $S^{2}$ of smaller and smaller radius. For an 'infinite' magnetic field, these circles become points, and the magnetic flow is along the fibres of $T^{*} S^{2}$.

In order to include this case, we consider the circular family of closed 2-forms

$$
\sin \theta \mathrm{d} \lambda+\cos \theta \pi^{*} \sigma_{0}, \quad \theta \in \mathbb{R} / 2 \pi \mathbb{Z} .
$$

For $\theta \equiv 0 \bmod \pi$, this form is not symplectic. There is, however, a simple way to extend $\pi^{*} \sigma_{0}$ to a symplectic form on $T^{*} S^{2} \backslash S^{2}$ (the complement of the zero section in $T^{*} S^{2}$ ) whose restriction to the tangent bundle of $S T^{*} S^{2}$ coincides with that of $\pi^{*} \sigma_{0}$. This is done as follows.

On $T^{*} S^{2}$ we have the canonical Liouville vector field $Y=Y_{T^{*} S^{2}}$ defined by $\mathrm{d} \lambda(Y,)=.\lambda$. In local coordinates $q_{1}, q_{2}$ on $S^{2}$ and dual coordinates $p_{1}, p_{2}$ on $T^{*} S^{2}$ we have $\lambda=p_{1} \mathrm{~d} q_{1}+p_{2} \mathrm{~d} q_{2}$, and hence $Y=p_{1} \partial_{p_{1}}+p_{2} \partial_{p_{2}}$ is the fibrewise radial vector field. We now identify $\mathbb{R}^{+} \times S T^{*} S^{2}$, where we write $\rho$ for the $\mathbb{R}^{+}$-coordinate, with $T^{*} S^{2} \backslash S^{2}$ by sending $\{1\} \times S T^{*} S^{2}$ identically to $S T^{*} S^{2}$, and the flow lines of $\rho \partial_{\rho}$ to the flow lines of $Y$. Under this identification, the symplectic form $\mathrm{d} \lambda$ on $T^{*} S^{2}$ pulls back to the symplectisation $\mathrm{d}\left(\rho \lambda_{1}\right)$ of the contact form $\lambda_{1}$ on $S T^{*} S^{2}$ given by the restriction of $\lambda$.

On the unit cotangent bundle $S T^{*} S^{2}$ we have a further contact form, namely, the connection 1-form $\alpha$, which satisfies $\mathrm{d} \alpha=\pi^{*} \sigma_{0}$; notice that the Euler class 
of the bundle is given by $-\left[\sigma_{0} / 2 \pi\right]=-2$. We then obtain a circular family of symplectic forms on $S T^{*} S^{2} \backslash S^{2} \equiv \mathbb{R}^{+} \times S T^{*} S^{2}$ by setting

$$
\omega^{\theta}:=\sin \theta \mathrm{d} \lambda+\cos \theta \mathrm{d}(\rho \alpha), \quad \theta \in \mathbb{R} / 2 \pi \mathbb{Z} .
$$

Remark 2.2. The Hamiltonian $H\left(\mathbf{v}^{*}\right)=\frac{1}{2}\left\|\mathbf{v}^{*}\right\|_{*}^{2}$ on $T^{*} S^{2}$ corresponds to the Hamiltonian $\rho^{2} / 2$ on $\mathbb{R}^{+} \times S T^{*} S^{2}$, for both functions take the value $1 / 2$ on $S T^{*} S^{2}$ and are homogeneous of degree 2 with respect to the flow of $Y \equiv \rho \partial_{\rho}$. It follows that the Hamiltonian flow of $\omega^{\theta}$ on $H^{-1}(1 / 2)=\{\rho=1\}$ coincides with the Reeb flow of the contact form $\sin \theta \lambda_{1}+\cos \theta \alpha$.

The Hamiltonian function $H$ is fixed throughout our discussion, so from now on we shall usually speak of 'the Hamiltonian flow of $\omega^{\theta}$ ' whenever the Hamiltonian $H$ is to be understood.

For $\theta \not \equiv 0 \bmod \pi$, the Hamiltonian flow of $\omega^{\theta}$ on the unit cotangent bundle $S T^{*} S^{2}=H^{-1}(1 / 2)$ equals the magnetic flow of $\left(g_{0},-\cot \theta \sigma_{0}\right)$, up to constant time reparametrisation; in particular, for $\theta \equiv \pi / 2 \bmod \pi$, the Hamiltonian flow is the geodesic flow of $g_{0}$. For $\theta \equiv 0 \bmod \pi$, the flow rotates the fibres of $S T^{*} S^{2}$.

There is a natural double covering of $S T^{*} S^{2}$ by the 3-sphere $S^{3}$ (Section 2.5), and a left-action of $S^{3}$ on itself coming from the multiplication of unit quaternions. The $S^{1}$-action mentioned in the following main theorem of this section comes from choosing a circle of unit quaternions (see Section 2.6). The contact forms $\alpha_{\mathbf{i}}, \alpha_{\mathbf{j}}$ in the theorem are the ones defined in the introduction.

Theorem 2.3. There are contact forms $\alpha_{\mathbf{i}}, \alpha_{\mathbf{j}}$ on $S^{3}$ and an $S^{1}$-action on $S^{3}$ sending $\alpha_{\mathbf{i}}$ to contact forms $\alpha^{\theta}=\cos \theta \alpha_{\mathbf{i}}+\sin \theta \alpha_{\mathbf{j}}, \theta \in \mathbb{R} / 2 \pi \mathbb{Z}$, such that the Reeb flow of $\alpha^{\theta}$, which has all orbits periodic of the same period $4 \pi$, doubly covers the Hamiltonian flow of $\omega^{\theta}$ on $S T^{*} S^{2}$. Moreover, there is a deformation $\alpha_{\mathbf{i}, \varepsilon}, \varepsilon \in[0,1)$, through contact forms, mapped to a family of contact forms $\alpha_{\varepsilon}^{\theta}$ under the $S^{1}$-action, with the following properties:

(i) The Reeb flow of $\alpha_{\varepsilon}^{\theta}$ doubly covers the Hamiltonian flow of $\omega^{\theta}$ on a level set of a suitably perturbed Hamiltonian function $H_{\varepsilon}^{\theta}$.

(ii) For any irrational value of the parameter $\varepsilon$, this Hamiltonian flow has precisely two periodic orbits.

(iii) For $\theta \equiv \pi / 2 \bmod \pi$ and $\varepsilon$ sufficiently small, the Hamiltonian flow of $H_{\varepsilon}^{\theta}$ is the geodesic flow of a Finsler metric on $S^{2}$.

Remark 2.4. There is in fact a whole $S^{2}$-family of such contact forms, with the corresponding deformations. For symmetry reasons, all the dynamical phenomena are present in the smaller $S^{1}$-family.

Theorem 2.3 is a variant of [2, Theorem 1.3]. The proof there hinges on a subtle construction of a family of symplectomorphisms

$$
\left(\left\{\left\|\mathbf{v}^{*}\right\|_{*}>s\right\}, \mathrm{d} \lambda\right) \longrightarrow\left(\left\{\left\|\mathbf{v}^{*}\right\|_{*}>0\right\}, \omega_{s \sigma_{0}}\right)
$$

for $s \in[0, \infty)$. In our proof, by contrast, the variation in the $s$-parameter (or the $\theta$-parameter in our set-up) becomes a perfectly straightforward application of a quaternionic symmetry, and the case $s=\infty$ is included naturally in the family.

Concerning the perturbation into Hamiltonian flows with only two periodic orbits, the essential feature of our argument is the following. Rather than perturbing the Hamiltonian function and the corresponding level sets, as the formulation of the theorem suggests, we actually deform the contact forms $\mathbb{Z}_{2}$-equivariantly on $S^{3}$, 
so that we always talk about Reeb flows on the fixed manifold $S T^{*} S^{2}$. Only then do we interpret these Reeb flows as Hamiltonian flows for a deformed Hamiltonian function. This approach via Reeb flows has the benefit that the quaternionic symmetry is preserved throughout the deformation, so the fact that the contact forms in the family $\left\{\alpha_{\varepsilon}^{\theta}\right\}_{\theta \in \mathbb{R} / 2 \pi \mathbb{Z}}$ share the same Reeb dynamics for any fixed value of the deformation parameter $\varepsilon$ becomes a tautology.

2.1. The structure forms on a surface. Let $\Sigma$ be an oriented surface with a Riemannian metric $g$ and associated complex structure $J$. Write $\pi: S T \Sigma \rightarrow \Sigma$ for the unit tangent bundle of $(\Sigma, g)$. The Liouville-Cartan forms of $(\Sigma, g)$ are the 1-forms $\lambda_{1}, \lambda_{2}$ on $S T \Sigma$ defined by the following equations, where $\mathbf{v} \in S T \Sigma$ and $\mathbf{t} \in T_{\mathbf{v}}(S T \Sigma):$

$$
\begin{aligned}
\lambda_{1}(\mathbf{t}) & =g\left(\mathbf{v}, T_{\mathbf{v}} \pi(\mathbf{t})\right), \\
\lambda_{2}(\mathbf{t}) & =g\left(J \mathbf{v}, T_{\mathbf{v}} \pi(\mathbf{t})\right)=-g\left(\mathbf{v}, J T_{\mathbf{v}} \pi(\mathbf{t})\right) .
\end{aligned}
$$

It is well known and easy to check, see [23, Section 7.2], that there is a unique 1 -form $\alpha_{*}$ on $S T \Sigma$ satisfying the structure equations

$$
\begin{aligned}
& \mathrm{d} \lambda_{1}=-\lambda_{2} \wedge \alpha_{*}, \\
& \mathrm{~d} \lambda_{2}=-\alpha_{*} \wedge \lambda_{1} .
\end{aligned}
$$

This 1-form $\alpha_{*}$ is the connection form on $S T \Sigma$, and the third structure equation is

$$
\mathrm{d} \alpha_{*}=-\left(\pi^{*} K\right) \lambda_{1} \wedge \lambda_{2},
$$

where $K$ is the Gauß curvature of $(\Sigma, g)$.

Remark 2.5. Pick any local orthonormal frame $\left(\mu_{1}, \mu_{2}\right)$ of 1 -forms on $(\Sigma, g)$. In a local trivialisation of the unit tangent bundle we may think of $\lambda_{1}, \lambda_{2}$ as

$$
\lambda_{1}=\cos \theta \mu_{1}+\sin \theta \mu_{2}, \quad \lambda_{2}=-\sin \theta \mu_{1}+\cos \theta \mu_{2} .
$$

The formula above then translates into a quick recipe for computing the Gauß curvature $K$ of $(\Sigma, g)$, see [15, Section 4.14]. Define local functions $a_{1}, a_{2}$ on $\Sigma$ by

$$
\mathrm{d} \mu_{1}=a_{1} \mu_{1} \wedge \mu_{2}, \quad \mathrm{~d} \mu_{2}=a_{2} \mu_{1} \wedge \mu_{2} .
$$

Set $\mu=-a_{1} \mu_{1}-a_{2} \mu_{2}$. Then $K$ is found via the equation $\mathrm{d} \mu=K \mu_{1} \wedge \mu_{2}$.

We also write $\lambda_{1}, \lambda_{2}$ for the corresponding 1-forms on the unit cotangent bundle $S T^{*} \Sigma$ under the bundle isomorphism $S T \Sigma \rightarrow S T^{*} \Sigma$ induced by the metric $g$. The natural orientation on the fibres of $S T^{*} \Sigma$ is the one for which this bundle isomorphism is fibre orientation reversing, since dual bundles have opposite Euler classes. So the connection 1-form $\alpha^{*}$ on $S T^{*} \Sigma$ corresponds to $-\alpha_{*}$. This means that the structure forms $\left(\lambda_{1}, \lambda_{2}, \alpha^{*}\right)$ on $S T^{*} \Sigma$ satisfy structure equations as on $S T \Sigma$, but with the minus signs in the three equations removed.

From now on we shall only be working on cotangent bundles, and we write the connection 1-form $\alpha^{*}$ simply as $\alpha$.

Remark 2.6. The 1-form $\lambda_{1}$ on $S T^{*} \Sigma$ is the restriction of the canonical Liouville 1form $\lambda$ on $T^{*} \Sigma$. If $q_{1}+q_{2} \mathbf{i}$ is a local holomorphic coordinate on $\Sigma$, and $p_{1}, p_{2}$ are the dual coordinates of $q_{1}, q_{2}$ on $T^{*} \Sigma$, then $\lambda_{1}=p_{1} \mathrm{~d} q_{1}+p_{2} \mathrm{~d} q_{2}$ and $\lambda_{2}=p_{1} \mathrm{~d} q_{2}-p_{2} \mathrm{~d} q_{1}$. 
2.2. The structure forms on $S^{2}$. Let $g_{0}$ be the round metric on $S^{2}$ of constant Gauß curvature 1, that is, the Riemannian metric induced on the unit sphere $S^{2} \subset$ $\mathbb{R}^{3}$ by the standard scalar product $\langle.,$.$\rangle on \mathbb{R}^{3}$. We may then think of the unit cotangent bundle $S T^{*} S^{2}$ as the submanifold

$$
S T^{*} S^{2}=\left\{(\mathbf{x}, \mathbf{y}) \in \mathbb{R}^{6}:|\mathbf{x}|=1,|\mathbf{y}|=1,\langle\mathbf{x}, \mathbf{y}\rangle=0\right\}
$$

of $\mathbb{R}^{6}$, with $(\mathbf{x}, \mathbf{y})$ interpreted as the covector $\dot{\mathbf{x}} \mapsto\langle\mathbf{y}, \dot{\mathbf{x}}\rangle$ for $\dot{\mathbf{x}} \in T_{\mathbf{x}} S^{2}$. Notice that with respect to the natural orientation of $S^{2}$, the positive orientation of $S T_{\mathrm{x}}^{*} S^{2}$ is defined by ordered pairs of covectors of the form $(\mathbf{x}, \mathbf{y})$ and $(\mathbf{x},-\mathbf{x} \times \mathbf{y})$.

The pair $\left(\lambda_{1}, \lambda_{2}\right)$ of Liouville-Cartan forms on $S T^{*} S^{2}$ in this model is given by

$$
\begin{aligned}
& \left(\lambda_{1}\right)_{(\mathbf{x}, \mathbf{y})}(\dot{\mathbf{x}}, \dot{\mathbf{y}})=\langle\mathbf{y}, \dot{\mathbf{x}}\rangle, \\
& \left(\lambda_{2}\right)_{(\mathbf{x}, \mathbf{y})}(\dot{\mathbf{x}}, \dot{\mathbf{y}})=\langle\mathbf{x} \times \mathbf{y}, \dot{\mathbf{x}}\rangle .
\end{aligned}
$$

With $\alpha$ denoting the connection 1-form on $S T^{*} S^{2}$ we have the structure equations

$$
\begin{aligned}
\mathrm{d} \lambda_{1} & =\lambda_{2} \wedge \alpha, \\
\mathrm{d} \lambda_{2} & =\alpha \wedge \lambda_{1}, \\
\mathrm{~d} \alpha & =\lambda_{1} \wedge \lambda_{2} .
\end{aligned}
$$

The last equation shows $\lambda_{1} \wedge \lambda_{2}$ to be invariant under the flow along the fibres of $\pi: S T^{*} S^{2} \rightarrow S^{2}$, and from the definition of the $\lambda_{i}$ we then see that $\lambda_{1} \wedge \lambda_{2}$ is the lift of the area form $\sigma_{0}$ on $S^{2}$ corresponding to the metric $g_{0}$, that is, $\mathrm{d} \alpha=\pi^{*} \sigma_{0}$.

2.3. Contact forms on $S^{3}$ induced by the quaternions. We regard $S^{3}$ as the unit sphere in the space $\mathbb{H} \cong \mathbb{R}^{4}$ of quaternions. We write $\mathbf{i}, \mathbf{j}, \mathbf{k}$ for the standard quaternionic units, and we use the same notation for the complex bundle structures they induce on the tangent bundle of $\mathbb{H}$. The units $\mathbf{i}, \mathbf{j}, \mathbf{k}$ define an identification of $\mathbb{R}^{3}$ with the space of pure imaginary quaternions. For other aspects of quaternionic notation see Appendix $\mathrm{A}$

Any element $\mathbf{c}=c_{1} \mathbf{i}+c_{2} \mathbf{j}+c_{3} \mathbf{k} \in S^{2} \subset \mathbb{R}^{3}$ defines a complex structure on $\mathbb{H}$, that is, $\mathbf{c}^{2}=-\mathrm{id}_{T \mathbb{H}}$. This gives rise to a 2 -sphere of contact forms

$$
\alpha_{\mathbf{c}}:=-\left.2 \mathrm{~d} r \circ \mathbf{c}\right|_{T S^{3}}, \quad \mathbf{c} \in S^{2},
$$

on $S^{3}$, where $r$ is the radial function on $\mathbb{H}$. The Reeb vector field of $\alpha_{\mathbf{c}}$ is

$$
R_{\mathbf{c}}=\frac{1}{2} \mathbf{c} \partial_{r} .
$$

For a general discussion of such 2-spheres of contact forms see [11, 13.

2.4. The Hopf fibration on $S^{3}$. The 2 -dimensional complex vector space $\mathbb{C}^{2}$ can be identified with the space $\mathbb{H}$ of quaternions via

$$
\mathbb{C}^{2} \ni\left(z_{0}, z_{1}\right) \longmapsto z_{0}+z_{1} \mathbf{j} \in \mathbb{H} .
$$

We consider the Hopf fibration corresponding to this choice of coordinates, that is,

$$
\mathbb{C}^{2} \supset S^{3} \ni\left(z_{0}, z_{1}\right) \longmapsto\left[z_{0}: z_{1}\right] \in \mathbb{C P}^{1}=S^{2} .
$$

The fibre of this Hopf fibration over the point $\left[z_{0}: z_{1}\right]$ is parametrised by $\mathrm{e}^{\mathbf{i} t / 2}\left(z_{0}, z_{1}\right)$, $t \in \mathbb{R} / 4 \pi \mathbb{Z}$. This choice of parametrisation corresponds to regarding the fibres as the orbits of the Reeb vector field $R_{\mathbf{i}}$. Notice that with $z_{0}=x_{0}+y_{0} \mathbf{i}, z_{1}=x_{1}+y_{1} \mathbf{i}$ we have

$$
\alpha_{\mathbf{i}}=2\left(x_{0} \mathrm{~d} y_{0}-y_{0} \mathrm{~d} x_{0}+x_{1} \mathrm{~d} y_{1}-y_{1} \mathrm{~d} x_{1}\right)
$$


and

$$
R_{\mathbf{i}}=\frac{1}{2}\left(x_{0} \partial_{y_{0}}-y_{0} \partial_{x_{0}}+x_{1} \partial_{y_{1}}-y_{1} \partial_{x_{1}}\right) .
$$

From the corresponding expressions for $\alpha_{\mathbf{j}}$ and $\alpha_{\mathbf{k}}$ it is easy to check that

$$
\begin{aligned}
\mathrm{d} \alpha_{\mathbf{i}} & =\alpha_{\mathbf{j}} \wedge \alpha_{\mathbf{k}}, \\
\mathrm{d} \alpha_{\mathbf{j}} & =\alpha_{\mathbf{k}} \wedge \alpha_{\mathbf{i}}, \\
\mathrm{d} \alpha_{\mathbf{k}} & =\alpha_{\mathbf{i}} \wedge \alpha_{\mathbf{k}} .
\end{aligned}
$$

In other words, the triple $\left(\alpha_{\mathbf{i}}, \alpha_{\mathbf{j}}, \alpha_{\mathbf{k}}\right)$ of 1-forms on $S^{3}$ satisfies the same structure equations as the triple $\left(\tilde{\alpha}, \tilde{\lambda}_{1}, \tilde{\lambda}_{2}\right)$ obtained by lifting the structure forms for the constant curvature 1 metric on $S^{2}$ from $S T^{*} S^{2}$ to $S^{3}$.

Here is a quick sketch, cf. [12, Section 3], how one may now argue that there is a diffeomorphism $S^{3} \rightarrow S^{3}$ that pulls back $\left(\tilde{\alpha}, \tilde{\lambda}_{1}, \tilde{\lambda}_{2}\right)$ to $\left(\alpha_{\mathbf{i}}, \alpha_{\mathbf{j}}, \alpha_{\mathbf{k}}\right)$; this diffeomorphism will be constructed explicitly in the next section.

Regard the 1 -forms $\alpha_{\mathbf{i}}-\tilde{\alpha}, \alpha_{\mathbf{j}}-\tilde{\lambda}_{1}, \alpha_{\mathbf{k}}-\tilde{\lambda}_{2}$ in the obvious way as 1 -forms on $S^{3} \times S^{3}$. The structure equations imply that this triple of 1 -forms generates a differential ideal, and hence defines a 3 -dimensional foliation on $S^{3} \times S^{3}$. Since either triple $\left(\tilde{\alpha}, \tilde{\lambda}_{1}, \tilde{\lambda}_{2}\right)$ and $\left(\alpha_{\mathbf{i}}, \alpha_{\mathbf{j}}, \alpha_{\mathbf{k}}\right)$ defines a coframe on $S^{3}$, the leaves of this foliation are graph-like with respect to either $S^{3}$-factor. Because of the compactness of $S^{3} \times S^{3}$, the inclusion of a leaf in $S^{3} \times S^{3}$, followed by the projection onto the first factor, is a covering. Since $S^{3}$ is simply connected, this covering must be trivial, so the leaves are indeed graphs of the desired diffeomorphism.

Since the 1-forms $\alpha_{\mathbf{c}}$ are invariant under the right-action by $S^{3}$ on itself, see Remark 2.13, this diffeomorphism is unique up to precomposition with the rightmultiplication by an element of $S^{3}$, cf. [12, Lemma 3.9].

2.5. The double covering $S^{3} \rightarrow S T^{*} S^{2}$. We think of $S^{3}$ as the unit sphere in $\mathbb{H}$, and we identify $\mathbb{R}^{3}$ with the space of pure imaginary quaternions. For $\mathbf{u} \in S^{3}$ and $\mathbf{x} \in \mathbb{R}^{3}$, define $f_{\mathbf{u}}(\mathbf{x}):=\overline{\mathbf{u}} \mathbf{x u}$. Then $f_{\mathbf{u}}$ is in fact an element of $\operatorname{SO}(3)$, and the map $\mathbf{u} \mapsto f_{\mathbf{u}}$ is a double covering $S^{3} \rightarrow \mathrm{SO}(3)$, see [10, Theorem 10.9].

Writing the elements of $\mathrm{SO}(3)$ in matrix form, we can describe the map $\mathbf{u} \mapsto f_{\mathbf{u}}$ more explicitly by

$$
\mathbf{u} \longmapsto\left(\begin{array}{ccc}
\mid & \mid & \mid \\
\mathbf{u} i u & \overline{\mathbf{u} j u} \mathbf{u} & \overline{\mathbf{u}} \mathbf{k u} \\
\mid & \mid & \mid
\end{array}\right)
$$

Remark 2.7. Notice that the last column is determined by the first two. In fact, for $\mathbf{x}, \mathbf{y} \in \mathbb{R}^{3} \subset \mathbb{H}$ one has

$$
\mathbf{x y}-\mathbf{y x}=2 \mathbf{x} \times \mathbf{y}
$$

see [10, Lemma 10.8]. One then checks easily that $\overline{\mathbf{u}} \mathbf{i u} \times \overline{\mathbf{u}} \mathbf{j u}=\overline{\mathbf{u}} \mathbf{k u}$.

Any two columns of a special orthogonal matrix define an element of $S T^{*} S^{2}$, with the model given in Section 2.2, and this gives a diffeomorphism from $\mathrm{SO}(3)$ to $S T^{*} S^{2}$. The following choice of double covering $S^{3} \rightarrow S T^{*} S^{2}$ is made so as to be compatible with the Hopf fibration and such that the structure forms on $S T^{*} S^{2}$ pull back to the triple of contact forms on $S^{3}$ induced by the quaternions. We first verify the statement about the Hopf fibration. 
Lemma 2.8. The covering

$$
\begin{aligned}
& \Phi: \quad S^{3} \longrightarrow S T^{*} S^{2} \subset \mathbb{R}^{6} \\
& \mathbf{u} \longmapsto \text { (uiu, } \overline{\mathbf{u}} \mathbf{k u}) \text {. }
\end{aligned}
$$

sends the Hopf fibres of $S^{3}$ two-to-one onto the fibres of $S T^{*} S^{2}$.

Proof. The Hopf fibres are the orbits of the $S^{1}$-action $\mathbf{u} \mapsto \mathrm{e}^{\mathbf{i} t / 2} \mathbf{u}, t \in \mathbb{R} / 4 \pi \mathbb{Z}$. Under this action we have

$$
\overline{\mathbf{u i u}} \longmapsto \overline{\mathbf{u}} \mathrm{e}^{-\mathrm{i} t / 2} \mathbf{i} \mathrm{e}^{\mathrm{i} t / 2} \mathbf{u}=\overline{\mathbf{u}} \mathbf{i u},
$$

which means that $\Phi$ is fibre-preserving. On the fibre coordinate of $S T^{*} S^{2}$ we have

$$
\overline{\mathbf{u}} \mathbf{k u} \longmapsto \overline{\mathbf{u}} \mathrm{e}^{-\mathbf{i} t / 2} \mathbf{k} \mathrm{e}^{\mathbf{i} t / 2} \mathbf{u}=\overline{\mathbf{u}}(\mathbf{j} \sin t+\mathbf{k} \cos t) \mathbf{u} .
$$

Since $t$ ranges over $\mathbb{R} / 4 \pi \mathbb{Z}$, each fibre of $S T^{*} S^{2}$ is doubly covered by a Hopf fibre. Notice that the orientation is positive with respect to the natural orientation of the fibres of $S T^{*} S^{2}$.

Remark 2.9. We have $f_{\mathbf{v}} \circ f_{\mathbf{u}}=f_{\mathbf{u v}}$. From the group-theoretic point of view it might be a little more natural to define the double covering $S^{3} \rightarrow \mathrm{SO}(3)$ by sending $\mathbf{u}$ to the map $\mathbf{x} \mapsto \mathbf{u x} \overline{\mathbf{u}}$, as was done in [10]. The preceding lemma explains why, in the present context, our definition of $f_{\mathbf{u}}$ is the preferred one.

Observe that $\Phi(\mathbf{u})=\Phi(-\mathbf{u})$, so $\Phi$ induces a diffeomorphism from $\mathbb{R P}^{3}$, the quotient of $S^{3}$ under the antipodal map, to $S T^{*} S^{2}$.

Remark 2.10. This lemma implies that $\Phi$ induces a diffeomorphism on $S^{2}$ (as the base of the Hopf fibration and of the unit cotangent bundle, respectively). We want to check that with respect to an appropriate choice of cartesian coordinates on $\mathbb{R}^{3}$ this map is the identity. We have

$$
\begin{aligned}
\overline{\mathbf{u i u}}= & \left(u_{0}^{2}+u_{1}^{2}-u_{2}^{2}-u_{3}^{2}\right) \mathbf{i}+2\left(u_{1} u_{2}-u_{0} u_{3}\right) \mathbf{j} \\
& +2\left(u_{0} u_{2}+u_{1} u_{3}\right) \mathbf{k} \\
= & : \quad x_{3} \mathbf{i}-x_{2} \mathbf{j}+x_{1} \mathbf{k} .
\end{aligned}
$$

For $S^{2} \subset \mathbb{R}_{x_{1}, x_{2}, x_{3}}^{3}$ consider the stereographic projection from the south pole $(0,0,-1)$ onto the equatorial $x_{1} x_{2}$-plane, which we identify with $\mathbb{C}$. Notice that both the chosen permutation of cartesian coordinates and this stereographic projection are orientation-preserving. As image of the stereographic projection we obtain

$$
\frac{x_{1}+x_{2} \mathbf{i}}{1+x_{3}}=\frac{\left(u_{0} u_{2}+u_{1} u_{3}\right)+\left(u_{0} u_{3}-u_{1} u_{2}\right) \mathbf{i}}{u_{0}^{2}+u_{1}^{2}}=\frac{u_{2}+u_{3} \mathbf{i}}{u_{0}+u_{1} \mathbf{i}},
$$

which equals $z_{1} / z_{0}$ under the identification of $\mathbf{u}$ with $z_{0}+z_{1} \mathbf{j}$.

The following lemma says that the structure forms pull back as desired.

Lemma 2.11. Let $\lambda_{1}, \lambda_{2}$ be the Liouville-Cartan forms on $S T^{*} S^{2}$, and $\alpha$ the connection 1-form. Then $\Phi^{*} \lambda_{1}=\alpha_{\mathbf{j}}, \Phi^{*} \lambda_{2}=\alpha_{\mathbf{k}}$, and $\Phi^{*} \alpha=\alpha_{\mathbf{i}}$.

Proof. The first two equalities are proved by a quaternionic computation that can be found in Appendix $\mathrm{\AA}$. The connection form $\alpha$ is determined by the structural equations for $\mathrm{d} \lambda_{1}$ and $\mathrm{d} \lambda_{2} ;$ likewise, $\alpha_{\mathbf{i}}$ is determined by $\alpha_{\mathbf{j}}$ and $\alpha_{\mathbf{k}}$. This yields the third equality. 
2.6. The quaternionic action. The unit quaternions $S^{3} \subset \mathbb{H}$ form a group, and we consider the left-action of $S^{3}$ on itself. For $\mathbf{a} \in S^{3}$ we write

$$
\begin{aligned}
l_{\mathbf{a}}: \quad S^{3} & \longrightarrow S^{3} \\
\mathbf{u} & \longmapsto \mathbf{a u} .
\end{aligned}
$$

Since we are ultimately concerned with Reeb orbits rather than contact forms, we formulate the following lemma in terms of the push-forward of forms.

Lemma 2.12. The push-forward of the contact form $\alpha_{\mathbf{c}}$ under $l_{\mathbf{a}}$ is the contact form $\alpha_{\mathbf{a c} \overline{\mathbf{a}}}$. In particular, we have $T l_{\mathbf{a}}\left(R_{\mathbf{c}}\right)=R_{\mathbf{a c} \overline{\mathbf{a}}}$.

Proof. The statement about the Reeb vector field is best proved directly. The orbits of $R_{\mathbf{c}}$ are described by the differential equation $\dot{\gamma}=\mathbf{c} \gamma / 2$. Then a $\gamma$ satisfies the differential equation

$$
\frac{\mathrm{d}}{\mathrm{d} t}(\mathbf{a} \gamma)=\mathbf{a} \dot{\gamma}=\frac{1}{2} \mathbf{a c} \gamma=\frac{1}{2}(\mathbf{a c} \overline{\mathbf{a}})(\mathbf{a} \gamma),
$$

so $\mathbf{a} \gamma$ is an orbit of $R_{\text {acc }}$.

For the transformation of the contact form, observe that for $\mathbf{p} \in S^{3}$ and $\mathbf{v} \in T_{\mathbf{p}} S^{3}$ we have $\mathrm{d} r_{\mathbf{p}}(\mathbf{c v})=\langle\mathbf{p}, \mathbf{c v}\rangle$, and use $l_{\mathbf{a}} \in \mathrm{SO}(4)$.

Remark 2.13. From $\mathrm{d} r_{\mathbf{p}}(\mathbf{c v})=\langle\mathbf{p}, \mathbf{c v}\rangle$ one also sees that the forms $\alpha_{\mathbf{c}}$ are invariant under the right-action of $S^{3}$ on itself.

We now take the Reeb flow of $\alpha_{\mathbf{i}}$, which defines the Hopf fibration, as our point of reference, and we want to understand how this flow transforms under the $S^{3}$ action. Notice that the transformation $\mathbf{i} \mapsto$ aia is the $\mathrm{SO}(3)$-action described in the preceding section. The 2 -sphere $S^{2} \subset \mathbb{R}^{3}$ of complex structures has an $S^{1}$-symmetry fixing $\mathbf{i}$, given by conjugation with $\mathbf{b}=\cos (\varphi / 2)+\mathbf{i} \sin (\varphi / 2)$. The left-action by $\mathbf{b}$ is simply a shift along the Hopf fibres. Thus, to understand the dynamics of $R_{\text {aia }}$, notably the projection of this flow to $S^{2}$, it suffices to consider a circular family of unit quaternions $\mathbf{a}$ for which ai $\mathbf{a}$ rotates in the $\mathbf{i j}$-plane, say. This is achieved by

in which case

$$
\mathbf{a}=\cos \frac{\theta}{2}+\mathbf{k} \sin \frac{\theta}{2}
$$

$$
\mathbf{a i} \overline{\mathbf{a}}=\mathbf{i} \cos \theta+\mathbf{j} \sin \theta .
$$

Proposition 2.14. For $\mathbf{a}=\cos (\theta / 2)+\sin (\theta / 2) \mathbf{k}, \theta \in] 0, \pi$, every orbit of $R_{\mathbf{a} i \overline{\mathbf{a}}}$ on $S^{3}$ projects under the Hopf fibration to a doubly covered circle of latitude of angle $\theta$ (measured from the pole defined by the corresponding orbit of $R_{\mathbf{i}}$ ).

Proof. For reasons of symmetry, it suffices to study the orbit of $R_{\mathrm{aia}}$ obtained via the transformation $l_{\mathbf{a}}$ from the orbit $\gamma$ of $R_{\mathbf{i}}$ (that is, the Hopf fibre) over the point $[1: 0] \in \mathbb{C P}^{1}$. The orbit $\gamma$ is parametrised by

$$
\gamma(t)=\left(\mathrm{e}^{\mathrm{i} t}, 0\right)=\left(\cos \frac{t}{2}, \sin \frac{t}{2}, 0,0\right), \quad t \in \mathbb{R} / 4 \pi \mathbb{Z} .
$$

The transformed orbit is

$$
\mathbf{a} \gamma(t)=\left(\cos \frac{\theta}{2} \cos \frac{t}{2}, \cos \frac{\theta}{2} \sin \frac{t}{2}, \sin \frac{\theta}{2} \sin \frac{t}{2}, \sin \frac{\theta}{2} \cos \frac{t}{2}\right) .
$$

This projects to $\mathbb{C P}^{1}$ as

$$
\left[\cos \frac{\theta}{2}\left(\cos \frac{t}{2}+\mathbf{i} \sin \frac{t}{2}\right): \mathbf{i} \sin \frac{\theta}{2}\left(\cos \frac{t}{2}-\mathbf{i} \sin \frac{t}{2}\right)\right]=\left[1: \mathbf{i e}^{-\mathbf{i} t} \tan \frac{\theta}{2}\right] .
$$


Figure 1 shows that this describes a circle of latitude of angle $\theta$ measured from the south pole $[1: 0]$. The circle is doubly covered, because $t$ ranges over $\mathbb{R} / 4 \pi \mathbb{Z}$.

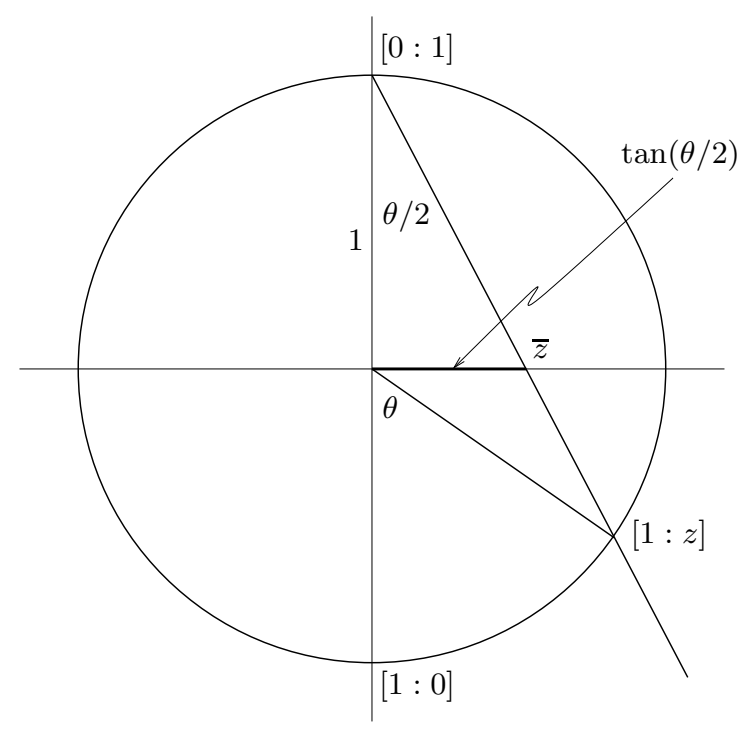

FIGURE 1. The circle of latitude of angle $\theta$.

Remark 2.15. For $\theta=\pi$, each Hopf fibre is mapped to the Hopf fibre over the respective antipodal point in $S^{2}$.

2.7. Magnetic flows. We now want to interpret the flow of the Reeb vector field $R_{\mathrm{ai} \overline{\mathrm{a}}}$ on $S^{3}$ as the lift of a magnetic flow on $S T^{*} S^{2}$. With a as in Proposition 2.14 we abbreviate $\alpha_{\text {aiā }}$ and $R_{\text {aia }}$ to $\alpha^{\theta}$ and $R^{\theta}$, respectively. With Lemma 2.11 we then have

$$
\alpha^{\theta}=\cos \theta \alpha_{\mathbf{i}}+\sin \theta \alpha_{\mathbf{j}}=\Phi^{*}\left(\cos \theta \alpha+\sin \theta \lambda_{1}\right) .
$$

Set

$$
\tilde{\omega}^{\theta}:=\mathrm{d} \alpha^{\theta}=\Phi^{*}\left(\cos \theta \pi^{*} \sigma_{0}+\sin \theta \mathrm{d} \lambda_{1}\right) .
$$

Remark 2.16. Notice that $\mathrm{d} \lambda_{1}$ extends naturally as a symplectic form on the full cotangent bundle as the canonical symplectic form $\mathrm{d} \lambda$. As discussed in the introduction to this Section 2 , we may also replace $\pi^{*} \sigma_{0}$ by the symplectic 2 -form $\mathrm{d}(\rho \alpha)$ defined on all of $T^{*} S^{2} \backslash S^{2}$. We may likewise think of $\tilde{\omega}^{\theta}$ as being defined on $\mathbb{R}^{4} \backslash\{0\} \cong \mathbb{R} \times S^{3}$ as the symplectisation of the contact form $\alpha^{\theta}$. Thus, we recognise $\tilde{\omega}^{\theta}$ as the double cover of the symplectic 2-form used in the definition of magnetic flows.

By Remark 2.2, the $S^{1}$-family of Reeb flows on $S^{3}$ defined by the contact forms $\left\{\alpha^{\theta}\right\}_{\theta \in \mathbb{R} / 2 \pi \mathbb{Z}}$ descends to $S T^{*} S^{2}=S^{3} / \mathbb{Z}_{2}$ to yield an interpolation via magnetic flows between the geodesic flow (for a vanishing magnetic term) and the Hopf flow along the fibres (for an 'infinite' magnetic field). 
2.8. Deformation of the magnetic flows. Recall from Section 2.4 the description of $\alpha_{\mathbf{i}}$ and $R_{\mathbf{i}}$ in terms of cartesian coordinates $\left(x_{0}, y_{0}, x_{1}, y_{1}\right)$. For $\varepsilon \in[0,1)$, we can consider the deformed contact form

$$
\alpha_{\mathbf{i}, \varepsilon}:=\frac{2}{1+\varepsilon}\left(x_{0} \mathrm{~d} y_{0}-y_{0} \mathrm{~d} x_{0}\right)+\frac{2}{1-\varepsilon}\left(x_{1} \mathrm{~d} y_{1}-y_{1} \mathrm{~d} x_{1}\right)
$$

on $S^{3}$, with Reeb vector field

$$
R_{\mathbf{i}, \varepsilon}=R_{\mathbf{i}}+\frac{\varepsilon}{2}\left(x_{0} \partial_{y_{0}}-y_{0} \partial_{x_{0}}\right)-\frac{\varepsilon}{2}\left(x_{1} \partial_{y_{1}}-y_{1} \partial_{x_{1}}\right)
$$

Notice that for irrational values of $\varepsilon$, with $(1+\varepsilon) /(1-\varepsilon)$ then likewise being irrational, the only periodic orbits of $R_{\mathbf{i}, \varepsilon}$ are $S^{3} \cap\left\{z_{1}=0\right\}$ and $S^{3} \cap\left\{z_{0}=0\right\}$, two circles forming a Hopf link. In other words, the two periodic orbits of $R_{\mathbf{i}}$ that persist after the deformation are the Hopf fibres over the points $[1: 0]$ and $[0: 1]$; the speed of the former increases, the latter is traversed more slowly.

Remark 2.17. The 1-form $\alpha_{\mathbf{i}, \varepsilon}$ on $S^{3}$ may be regarded as the contact form induced by $2 \sum_{i=0}^{1}\left(x_{i} \mathrm{~d} y_{i}-y_{i} \mathrm{~d} x_{i}\right)$ (regarded as a form on $\mathbb{R}^{4}$ ) under the embedding of $S^{3}$ as an ellipsoid $E_{\varepsilon}$, in complex notation,

$$
\begin{array}{ccc}
S^{3} & \longrightarrow & \left\{(1+\varepsilon)\left|z_{0}\right|^{2}+(1-\varepsilon)\left|z_{1}\right|^{2}=1\right\}=: E_{\varepsilon} \\
\left(z_{0}, z_{1}\right) & \longmapsto & \left(\frac{z_{0}}{\sqrt{1+\varepsilon}}, \frac{z_{1}}{\sqrt{1-\varepsilon}}\right) .
\end{array}
$$

It is useful, as in 25], to study the flow of $R_{\mathbf{i}}$ and the additional summand in $R_{\mathbf{i}, \varepsilon}$ separately. The flow

$$
t \longmapsto \mathrm{e}^{\mathrm{i} t / 2}\left(z_{0}, z_{1}\right)
$$

of $R_{\mathbf{i}}$ gives us the fibres of the Hopf fibration; the flow lines of

$$
\frac{1}{2}\left(x_{0} \partial_{y_{0}}-y_{0} \partial_{x_{0}}\right)-\frac{1}{2}\left(x_{1} \partial_{y_{1}}-y_{1} \partial_{x_{1}}\right)
$$

are parametrised by

$$
s \longmapsto\left(\mathrm{e}^{\mathrm{i} s / 2} z_{0}, \mathrm{e}^{-\mathrm{i} s / 2} z_{1}\right) .
$$

These two flows commute and define a $T^{2}$-action on $S^{3}$. The $\mathbb{R}$-action defined by the flow of $R_{\mathbf{i}, \varepsilon}$ corresponds to a line in the 2-torus $T^{2}=\mathbb{R}^{2} /(4 \pi \mathbb{Z})^{2}$ of slope $\varepsilon$.

The following geometric observation is not, strictly speaking, relevant to our discussion, but it is worth noting nonetheless.

Lemma 2.18. Let $\beta(s)=\left(\mathrm{e}^{\mathrm{i} s / 2} z_{0}, \mathrm{e}^{-\mathbf{i} s / 2} z_{1}\right), s \in \mathbb{R} / 4 \pi \mathbb{Z}$, be a flow line of the anti-diagonal $S^{1}$-action. For any $\mathbf{a} \in S^{3}$, the rotated flow line $l_{\mathbf{a}}(\beta)$ projects under the Hopf fibration to a circle of latitude on $S^{2}$ relative to the poles [0:1] and [1:0].

Proof. Write $\mathbf{a}=w_{0}+w_{1} \mathbf{j}$ with $w_{0}, w_{1} \in \mathbb{C}$. We compute

$$
\left(w_{0}+w_{1} \mathbf{j}\right)\left(\mathrm{e}^{\mathbf{i} s / 2} z_{0}+\mathrm{e}^{-\mathbf{i} s / 2} z_{1} \mathbf{j}\right)=\left(w_{0} z_{0}-w_{1} \bar{z}_{1}\right) \mathrm{e}^{\mathbf{i} s / 2}+\left(w_{0} z_{1}+w_{1} \bar{z}_{0}\right) \mathrm{e}^{-\mathbf{i} s / 2} .
$$

This projects to

$$
\left[1: \frac{w_{0} z_{1}+w_{1} \bar{z}_{0}}{w_{0} z_{0}-w_{1} \bar{z}_{1}} \mathrm{e}^{-\mathbf{i} s}\right],
$$

which describes a circle of latitude on $S^{2}$ as in the proof of Proposition 2.14, see also Figure 1. 
2.9. Proof of Theorem 2.3. With $\mathbf{a}=\cos (\theta / 2)+\sin (\theta / 2) \mathbf{k}$ as in Proposition 2.14 and Section 2.7 we set $\alpha_{\varepsilon}^{\theta}:=l_{\mathbf{a}}\left(\alpha_{\mathbf{i}, \varepsilon}\right)$. As we have seen, the Reeb flow of $\alpha_{\mathbf{i}, \varepsilon}$ has precisely two periodic orbits for any irrational value of the deformation parameter $\varepsilon$. Since the Reeb flow of $\alpha_{\mathbf{i}, \varepsilon}$ is equivariant with respect to the antipodal map, the induced flow on $S T^{*} S^{2}$ likewise has precisely two periodic orbits, both doubly covered by the corresponding orbit on $S^{3}$. This proves part (ii) of the theorem, once the Hamiltonian interpretation in part (i) has been established.

In order to prove part (i), we may work on $\left(\mathbb{R}^{4} \backslash\{0\}, \tilde{\omega}^{\theta}\right)$, viewed as the symplectisation of $\left(S^{3}, \alpha^{\theta}\right)$, cf. Section 2.7 provided we ensure that our construction is $\mathbb{Z}_{2}$-invariant. Since the left-action of $S^{3}$ on itself extends to an action on $\mathbb{R}^{4} \backslash\{0\}$, it suffices to study the deformation $\alpha_{\mathbf{i}, \varepsilon}$ of $\alpha_{\mathbf{i}}$.

On $\mathbb{R}^{4} \backslash\{0\}=\mathbb{C}^{2} \backslash\{0\}$ with the symplectic form

$$
\tilde{\omega}^{0}=4\left(\mathrm{~d} x_{0} \wedge \mathrm{d} y_{0}+\mathrm{d} x_{1} \wedge \mathrm{d} y_{1}\right)
$$

we consider the Hamiltonian functions

$$
H_{0}\left(z_{0}, z_{1}\right):=\left|z_{0}\right|^{2}+\left|z_{1}\right|^{2} \text { and } F\left(z_{0}, z_{1}\right):=\left|z_{0}\right|^{2}-\left|z_{1}\right|^{2},
$$

and we set $H_{\varepsilon}:=H_{0}+\varepsilon F$. The Hamiltonian vector field of $H_{\varepsilon}$ is

$$
X_{\varepsilon}=\frac{1+\varepsilon}{2}\left(x_{0} \partial_{y_{0}}-y_{0} \partial_{x_{0}}\right)+\frac{1-\varepsilon}{2}\left(x_{1} \partial_{y_{1}}-y_{1} \partial_{x_{1}}\right) .
$$

So we recognise the Reeb vector field $R_{\mathbf{i}, \varepsilon}$ from Section 2.8 as the Hamiltonian vector field of $X_{\varepsilon}$ restricted to the level set $H_{\varepsilon}^{-1}(1)=E_{\varepsilon}$. Notice that the form of $X_{\varepsilon}$ does not change under the map that sends $S^{3}$ to $E_{\varepsilon}$.

Finally, we want to prove part (iii). It suffices to consider $\theta=\pi / 2$. Recall that $\alpha^{\pi / 2}=\alpha_{\mathbf{j}}$. We may work in the symplectic manifold

$$
\left(\mathbb{R}^{4} \backslash\{0\}, \tilde{\omega}^{\pi / 2}=2\left(\mathrm{~d} x_{1} \wedge \mathrm{d} x_{2}+\mathrm{d} y_{2} \wedge \mathrm{d} y_{1}\right)\right) .
$$

Under the left-action of $\mathbf{a} \in S^{3}$, the Hamiltonian function $H_{\varepsilon}$ transforms to the function $H_{\varepsilon} \circ l_{\mathbf{a}}^{-1}=H_{\varepsilon} \circ l_{\overline{\mathbf{a}}}$. The original Hamiltonian function $H_{0}$ is obviously invariant, since $l_{\mathbf{a}}$ is norm-preserving. The angle $\theta=\pi / 2$ corresponds to the quaternion $\mathbf{a}=\sqrt{2} / 2+\mathbf{k} \sqrt{2} / 2$. We compute

$$
\begin{aligned}
& \left(\frac{\sqrt{2}}{2}-\frac{\sqrt{2}}{2} \mathbf{k}\right) \cdot\left(x_{0}+y_{0} \mathbf{i}+x_{1} \mathbf{j}+y_{1} \mathbf{k}\right)= \\
& \left(\frac{\sqrt{2}}{2} x_{0}+\frac{\sqrt{2}}{2} y_{1}\right)+\left(\frac{\sqrt{2}}{2} y_{0}+\frac{\sqrt{2}}{2} x_{1}\right) \mathbf{i}+ \\
& \left(\frac{\sqrt{2}}{2} x_{1}-\frac{\sqrt{2}}{2} y_{0}\right) \mathbf{j}+\left(\frac{\sqrt{2}}{2} y_{1}-\frac{\sqrt{2}}{2} x_{0}\right) \mathbf{k} .
\end{aligned}
$$

This yields

$$
F \circ l_{\overline{\mathbf{a}}}\left(x_{0}, y_{0}, x_{1}, y_{1}\right)=x_{0} y_{1}+y_{0} x_{1},
$$

hence $H_{\varepsilon}$ transforms to

$$
\begin{aligned}
K_{\varepsilon}\left(x_{0}, y_{0}, x_{1}, y_{1}\right) & :=H_{\varepsilon} \circ l_{\overline{\mathbf{a}}}\left(x_{0}, y_{0}, x_{1}, y_{1}\right) \\
& =H_{0}\left(x_{0}, y_{0}, x_{1}, y_{1}\right)+\varepsilon\left(x_{0} y_{1}+y_{0} x_{1}\right) .
\end{aligned}
$$

In the notation of the theorem we have $K_{\varepsilon}=H_{\varepsilon}^{\pi / 2}$.

The following homogeneity property will be essential for the interpretation of the Hamiltonian flow of $K_{\varepsilon}$ as a Finsler geodesic flow. 
Lemma 2.19. When viewed as a function on $T^{*} S^{2}$, the Hamiltonian function $K_{\varepsilon}$ is homogeneous of degree 1 in the fibre coordinates.

Proof. The strict contactomorphism between $\left(S^{3}, \alpha_{\mathbf{j}}\right)$ and the double cover of the unit cotangent bundle $\left(S T^{*} S^{2}, \lambda_{1}\right)$ extends to a symplectomorphism

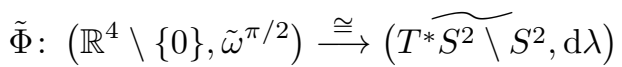

by sending the flow lines of the Liouville vector field

$$
Y_{\mathbb{R}^{4}}=\frac{1}{2}\left(x_{0} \partial_{x_{0}}+y_{0} \partial_{y_{0}}+x_{1} \partial_{x_{1}}+y_{1} \partial_{y_{1}}\right)
$$

for $\tilde{\omega}^{\pi / 2}$ to those of the fibrewise radial Liouville vector field $Y_{T^{*} S^{2}}$ (see the discussion before Remark 2.2), lifted to the double cover.

A function $G: T^{*} S^{2} \rightarrow \mathbb{R}$ is homogeneous of degree 1 in the fibre coordinates precisely if $\mathrm{d} G\left(Y_{T^{*} S^{2}}\right)=G$. Then the pulled-back function $G \circ \tilde{\Phi}$ satisfies the equation

$$
\mathrm{d}(G \circ \tilde{\Phi})\left(Y_{\mathbb{R}^{4}}\right)=G \circ \tilde{\Phi} .
$$

By Euler's theorem, because of the factor $1 / 2$ in $Y_{\mathbb{R}^{4}}$, this means that $G \circ \tilde{\Phi}$ is homogeneous of degree 2 in $\left(x_{0}, y_{0}, x_{1}, y_{1}\right)$. Conversely, a homogeneous function on $\mathbb{R}^{4}$ is mapped to a fibrewise homogeneous function on $T^{*} S^{2}$ of half the degree of homogeneity.

Since $K_{\varepsilon}$ is homogeneous of degree 2 on $\mathbb{R}^{4}$, this proves the lemma.

Remark 2.20. Since $H_{0}$ takes the value 1 on $S^{3}$, the corresponding Hamiltonian function on $T^{*} S^{2}$ is $\mathbf{v}^{*} \mapsto\left\|\mathbf{v}^{*}\right\|_{*}$.

As observed in Remark 2.1, the Hamiltonian flow of $H_{0}^{2} / 2$ is the geodesic flow. The Hamiltonian flow of $H_{0}$ is simply a reparametrisation of it, and on $S T^{*} S^{2}$ the two flows coincide, since $X_{H_{0}^{2} / 2}=H_{0}^{-1} X_{H_{0}}$. Similarly, the Hamiltonian flow of the function $K_{\varepsilon}$ is just a reparametrisation of the one defined by its square. The latter is fibrewise homogeneous of degree 2 and, for $\varepsilon=0$, fibrewise strictly convex. This condition is preserved for small values of $\varepsilon$, in which case $\left(K_{\varepsilon}\right)^{2}$ gives rise to a Finsler metric, see [25, p. 137] or [8].

This completes the proof of Theorem 2.3 .

Remark 2.21. (1) In the Finsler case $(\theta=\pi / 2)$, the two geodesics that survive the perturbation equal the great circle on $S^{2}$ with respect to the poles [1:0] and $[0: 1]$, traversed in opposite directions (and with different speeds). Since the Finsler metric is non-reversible, this does indeed count as two geodesics.

(2) If we label the coordinates on $\mathbb{R}^{4}=\mathbb{H}$ as

$$
\left(u_{0}, u_{1}, u_{2}, u_{3}\right) \text { instead of }\left(x_{0}, y_{0}, x_{1}, y_{1}\right),
$$

the perturbation of the Hamiltonian function in the Finsler case is described by $\varepsilon\left(u_{0} u_{3}+u_{1} u_{2}\right)$. Computing with the conventions for cartesian coordinates as in Remark 2.10, one finds with $\mathbf{x}=\overline{\mathbf{u i u}}$ and $\mathbf{y}=\overline{\mathbf{u}} \mathbf{k u}$ that

$$
-2\left(u_{0} u_{3}+u_{1} u_{2}\right)=x_{1} y_{2}-x_{2} y_{1} .
$$

This means that on $S T^{*} S^{2}$ the perturbation term is described by

$$
-\frac{\varepsilon}{2}\left\langle\left(y_{1}, y_{2}, y_{3}\right),\left(0,-x_{3}, x_{2}\right)\right\rangle \text {. }
$$


Since this expression is homogeneous of degree 1 in the fibre coordinates, it describes the perturbation term on the full cotangent bundle $T^{*} S^{2}$. Thus, up to a constant scale, the perturbation term is given by the function which sends a point in $T^{*} S^{2}$, i.e. a covector, to its evaluation on the vector field defining the rotation of $S^{2}$ about the $x_{1}$-axis. This is precisely the type of perturbation in Katok's example as described in [25, p. 137].

(3) Observe that the perturbation term, when interpreted dually on the tangent bundle $T S^{2}$, is given by a 1 -form $\mu$ on $S^{2}$ evaluated on tangent vectors, that is, the Finsler norm of $\mathbf{v} \in T_{\mathbf{x}} S^{2}$ is $\sqrt{g_{0, \mathbf{x}}(\mathbf{v}, \mathbf{v})}+\mu_{\mathbf{x}}(\mathbf{v})$. Finsler metrics of this type are called Randers metrics.

\section{ReEB Flows With A FINITE NUMBER OF PERIODIC ORBITS}

The example in Section 2.8 of a contact form on $S^{3}$ with precisely two periodic Reeb orbits easily generalises to higher dimensions. Given rationally independent positive real numbers $a_{0}, \ldots, a_{n}$, the 1 -form

$$
\sum_{i=0}^{n} a_{i}\left(x_{i} \mathrm{~d} y_{i}-y_{i} \mathrm{~d} x_{i}\right)
$$

on $\mathbb{R}^{2 n+2}$ induces a contact form on the unit sphere $S^{2 n+1} \subset \mathbb{R}^{2 n+2}$ whose periodic Reeb orbits are given by the intersection of that sphere with one of the $x_{i} y_{i}$-planes. So there are precisely $n+1$ periodic Reeb orbits. Conjecturally, this is the smallest possible number of periodic Reeb orbits in the given dimension.

Our aim in this section is to describe contact manifolds with a finite number of periodic Reeb orbits. In dimension three, there are some deep results concerning this issue. Taubes 24] has shown the existence of at least one periodic Reeb orbit on any closed contact 3-manifold, thus giving a positive answer to the Weinstein conjecture in this dimension. This has been extended by Cristofaro-Gardiner and Hutchings [6], who have shown that there will always be at least two periodic Reeb orbits.

It is an open question whether any contact form on a closed, connected 3manifold that has more than two periodic Reeb orbits actually possesses infinitely many of them. Under some additional assumptions, a positive answer to this question has recently been given by Cristofaro-Gardiner, Hutchings and Pomerleano [7. Recall that a contact form is called non-degenerate if the linearised return map at any periodic Reeb orbit does not have 1 as an eigenvalue.

Theorem 3.1 (Cristofaro-Gardiner, Hutchings, Pomerleano). Let $M$ be a closed, connected 3-manifold with a non-degenerate contact form $\alpha$. Assume further that the Euler class of the contact structure is a torsion element in $H^{2}(M ; \mathbb{Z})$. Then $\alpha$ has either two or infinitely many periodic Reeb orbits.

Combining this with a result of Hutchings and Taubes, it follows that under the assumptions of non-degeneracy and the Euler class being torsion, contact forms with precisely two periodic Reeb orbits exist only on $S^{3}$ and lens spaces.

Here we show that the situation in higher dimensions is completely different. There are examples of closed, connected contact manifolds with high finite numbers of periodic Reeb orbits.

We also consider closed characteristics on hypersurfaces in symplectic manifolds. Recall that the characteristics of a hypersurface $M$ in a symplectic manifold $(W, \omega)$ 
are the integral curves of $\operatorname{ker}\left(\left.\omega\right|_{T M}\right)$. The characteristics may also be regarded as the orbits of the Hamiltonian flow defined by any Hamiltonian function on $W$ having $M$ as a level set. We write $\omega_{\text {st }}=\sum_{i=1}^{n+1} \mathrm{~d} x_{i} \wedge \mathrm{d} y_{i}$ for the standard symplectic form on $\mathbb{R}^{2 n+2}$.

Theorem 3.2. (a) In any dimension $2 n+1 \geq 5$, there are closed, connected contact manifolds with arbitrarily large finite numbers of periodic Reeb orbits. In dimension five, for instance, any number $\geq 3$ can be so realised.

(b) For any natural number $n \geq 2$ and any non-negative integer $k$ there is a closed, connected hypersurface in $\mathbb{R}^{2 n+2}$ with precisely $k$ closed characteristics. For $n=1$, any number $k \geq 2$ can be realised.

Remark 3.3. Part (b) has also been proved, using more intricate arguments, by Cieliebak [5, Corollary J]. In fact, Cieliebak shows that for $k \geq 2$ closed characteristics, the hypersurface may be taken to be of so-called confoliation type.

3.1. Boothby-Wang bundles. We begin by considering contact forms on principal $S^{1}$-bundles that arise from the Boothby-Wang construction [3], cf. [9, Section 7.2]. Thus, we assume that $B$ is a closed manifold with a symplectic form $\omega$ such that the de Rham cohomology class $-[\omega / 2 \pi] \in H_{\mathrm{dR}}^{2}(B)$ is integral, i.e. it lies in the image of the homomorphism $H^{2}(B ; \mathbb{Z}) \rightarrow H^{2}(B ; \mathbb{R})=H_{\mathrm{dR}}^{2}(B)$ induced by the inclusion $\mathbb{Z} \rightarrow \mathbb{R}$ of coefficients. One can then find a connection 1 -form $\alpha$ on the principal $S^{1}$-bundle $\pi: M \rightarrow B$ of Euler class $-[\omega / 2 \pi]$ with curvature form $\omega$, that is, $\mathrm{d} \alpha=\pi^{*} \omega$. The assumption that $\omega$ be symplectic then translates into $\alpha$ being a contact form. We write $R$ for the Reeb vector field of $\alpha$. The orbits of $R$ are the fibres of the $S^{1}$-bundle. Our normalisation of the curvature form corresponds to regarding $S^{1}$ as $\mathbb{R} / 2 \pi \mathbb{Z}$.

3.2. Lifting Hamiltonian vector fields. Consider a smooth function $H: B \rightarrow \mathbb{R}$ and its Hamiltonian vector field $X=X_{H}$ with respect to the symplectic form $\omega$. Our aim is to lift $X$ to a Hamiltonian vector field $\tilde{X}$ with respect to the contact form $\alpha$ on $M$. This can be done in such a way that the corresponding contact Hamiltonian is invariant in the $R$-direction, so that the flow of $\tilde{X}$ preserves the contact form $\alpha$, not only the contact structure $\operatorname{ker} \alpha$.

Lemma 3.4. Let $X_{\mathrm{h}}$ be the horizontal lift of $X$, that is, the vector field on $M$ with $\alpha\left(X_{\mathrm{h}}\right)=0$ and $T \pi\left(X_{\mathrm{h}}\right)=X$, and let $\tilde{H}:=H \circ \pi: M \rightarrow \mathbb{R}$ be the lift of $H$. Then the flow of the vector field $\tilde{X}:=\tilde{H} R+X_{\mathrm{h}}$ preserves $\alpha$. If $H$ (and hence $\tilde{H}$ ) takes positive values only, the lifted vector field $\tilde{X}$ is the Reeb vector field of the rescaled contact form $\alpha / \tilde{H}$.

Proof. We compute the Lie derivative, writing $\iota$ for the interior product:

$$
\begin{aligned}
L_{\tilde{X}} \alpha & =\mathrm{d}(\alpha(\tilde{X}))+\iota_{\tilde{X}} \mathrm{~d} \alpha \\
& =\mathrm{d} \tilde{H}+\iota_{X_{\mathrm{h}}} \mathrm{d} \alpha \\
& =\mathrm{d} \tilde{H}+\pi^{*}\left(\iota_{X} \omega\right) \\
& =0,
\end{aligned}
$$

which proves the invariance of $\alpha$ under the flow of $\tilde{X}$. The statement about $\tilde{X}$ being the Reeb vector field of $\alpha / \tilde{H}$ in the case $\tilde{H}>0$ follows from the theory of contact Hamiltonians [9, Section 2.3], since the flow of $\tilde{X}$ preserves the contact structure $\operatorname{ker}(\alpha / \tilde{H})$, and $\tilde{X}$ evaluates to 1 on this rescaled contact form. 
Remark 3.5. Adding a constant $c \in \mathbb{R}$ to $H$ (and hence $\tilde{H}$ ) does not change $X$ or its horizontal lift $X_{\mathrm{h}}$; the lifted Hamiltonian vector field $\tilde{X}$ changes by $c R$. In this way we obtain all possible lifts of $X$ to a vector field whose flow preserves $\alpha$. By such a change we can always achieve $\tilde{H}>0$, since $B$ is assumed to be closed.

3.3. Lifting Hamiltonian $S^{1}$-actions. We now suppose that the Hamiltonian vector field $X=X_{H}$ on $(B, \omega)$ induces an action by the circle $S^{1}=\mathbb{R} / 2 \pi \mathbb{Z}$. We normalise $H$ by adding a constant in such a way that $H>0$ and, at some chosen singularity $p_{0}$ of $X$, the value $H\left(p_{0}\right)$ is a natural number. The following argument for showing that the lifted vector field $\tilde{X}$ likewise induces an $S^{1}$-action is similar to the one used in 21.

Proposition 3.6. A Hamiltonian $S^{1}$-action on $(B, \omega)$ lifts to a Hamiltonian $S^{1}$ action on the total space $(M, \alpha)$ of the Boothby-Wang bundle.

Proof. Over a singularity $p \in B$ of the vector field $X$ defining the $S^{1}$-action on $B$, the lifted vector field $\tilde{X}$ equals $H(p) R$, so the $\tilde{X}$-orbit through any point in the fibre $\pi^{-1}(p)$ is precisely that fibre, traversed positively. For $p=p_{0}$, the fibre is traversed $H\left(p_{0}\right)$ times. In order for the $\tilde{X}$-flow along the fibre over any other singularity $p$ to define an $S^{1}$-action, the value $H(p)$ has to be integral. This requirement is indeed satisfied, as the following argument shows.

Choose a smooth path $\gamma:[0,1] \rightarrow B$ from $\gamma(0)=p_{0}$ to $\gamma(1)=p$. By acting on this path with the Hamiltonian $S^{1}$-action, we obtain a 2 -sphere $S \subset B$, which we orient by the ordered frame $\dot{\gamma}, X$. Here 'sphere' is understood in the sense of smooth singular theory, see [4, Section V.5]. We then compute

$$
\begin{aligned}
H(p)-H\left(p_{0}\right) & =\int_{\gamma} \mathrm{d} H=-\int_{\gamma}\left(\iota_{X} \omega\right) \\
& =\frac{1}{2 \pi} \int_{S} \omega=\langle[\omega / 2 \pi],[S]\rangle,
\end{aligned}
$$

which is, up to sign, simply the evaluation of the Euler class on the homology class $[S]$, and hence an integer.

For any non-singular point $q \in B$ of $X$ we consider the $S^{1}$-orbit $\beta$ through $q$, which is a circle in $B$, perhaps multiply covered. We need to show that the $\tilde{X}$-path $\tilde{\beta}$ over $\beta$ is likewise a closed loop. By choosing a path $\gamma$ from $p_{0}$ to $q$ and acting on it as before, we create a disc $\Delta$ in $B$ with boundary $\beta$, again in the sense of smooth singular theory.

The horizontal lift $\beta_{\mathrm{h}}$ of $\beta$ starts at some point $\tilde{q}$ in the $S^{1}$-fibre over $q$ and ends at $\mathrm{e}^{\mathrm{i} h(\beta)}$, where $h(\beta)$ denotes the holonomy of $\beta$. This is computed as $h(\beta)=$ $-\int_{\Delta} \omega$, as can be seen either by applying the theorem of Stokes to a lifted disc $\tilde{\Delta}$ (with a boundary segment of oriented length $-h(\beta)$ lying in the fibre over $q$ ) or by computing explicitly in a trivialisation of the $S^{1}$-bundle over $\Delta$. Notice that the condition that $-[\omega / 2 \pi]$ be an integral cohomology class guarantees that, modulo $2 \pi$, the holonomy does not depend on the choice of disc $\Delta$ bounded by $\beta$, since the evaluation of $\omega$ over any 2-sphere made up of two such discs (one with reversed orientation) lies in $2 \pi \mathbb{Z}$.

Now, observing that the positive orientation of $\Delta$ (for the boundary orientation defined by $X$ ) is defined by the ordered frame $\dot{\gamma}, X$, we essentially perform the 
previous computation backwards:

$$
\begin{aligned}
h(\beta)=-\int_{\Delta} \omega & =2 \pi \int_{\gamma} \iota_{X} \omega \\
& =-2 \pi \int_{\gamma} \mathrm{d} H \\
& =-2 \pi\left(H(q)-H\left(p_{0}\right)\right) \equiv-2 \pi H(q) \bmod 2 \pi .
\end{aligned}
$$

On the other hand, along $\beta$ the Hamiltonian function takes the constant value $H(q)$, so the contribution of the $R$-component of $\tilde{X}$ to the flow over a period $2 \pi$ is $2 \pi H(q)$, which cancels the holonomy. This shows that $\tilde{\beta}$ is indeed a smooth loop.

Remark 3.7. There are far more general results about the lifting of group actions to principal bundles. The lifting of Hamiltonian group actions to prequantisation line bundles is discussed in [16], see in particular Theorem 6.7 and Example 6.10 there, and [22, Corollary 1.3].

3.4. A torus action on the Boothby-Wang bundle. We assume that we are in the situation of the preceding section. Apart from the $S^{1}$-action on $(M, \alpha)$ lifted from the Hamiltonian circle action on $(B, \omega)$, we also have the action given by the Reeb flow along the fibres. We have already seen that $L_{\tilde{X}} \alpha=0$; the condition $L_{R} \alpha=0$ is immediate from the defining equations of the Reeb vector field.

Lemma 3.8. The flows of $\tilde{X}=\tilde{H} R+X_{\mathrm{h}}$ and $R$ on $M$ commute and hence define a torus action preserving the contact form $\alpha$.

Proof. We show that $R$ commutes with the horizontal lift $X_{\mathrm{h}}$ of $X$. Since $\tilde{H}$ is constant along the fibres of the Boothby-Wang bundle, i.e. the $R$-orbits, the Reeb vector field then also commutes with $\tilde{X}$. From

$$
\alpha\left(\left[R, X_{\mathrm{h}}\right]\right)=\alpha\left(L_{R} X_{\mathrm{h}}\right)=L_{R}\left(\alpha\left(X_{\mathrm{h}}\right)\right)-\left(L_{R} \alpha\right)\left(X_{\mathrm{h}}\right)=0
$$

we see that $\left[R, X_{\mathrm{h}}\right]$ is horizontal. But also

$$
T \pi\left(\left[R, X_{\mathrm{h}}\right]\right)=\left[T \pi(R), T \pi\left(X_{\mathrm{h}}\right)\right]=0,
$$

which means that the Lie bracket $\left[R, X_{\mathrm{h}}\right]$ vanishes.

Provided the $S^{1}$-action on $B$ has finitely many fixed points, we can find a Reeb flow on $M$ with finitely many periodic orbits by choosing a line of irrational slope in the torus acting on $M$, analogous to the argument in Section 2.8 .

Proposition 3.9. If the Hamiltonian $S^{1}$-action on $(B, \omega)$ has finitely many fixed points, a suitably rescaled contact form on the Boothby-Wang bundle over $(B, \omega)$ has finitely many periodic Reeb orbits, namely, the fibres over the fixed points.

Proof. For $\varepsilon>0$ an irrational number, the flow of $\tilde{X}_{\varepsilon}:=\tilde{X}+\varepsilon R$ preserves $\alpha$, and its only periodic orbits are the fibres over the fixed points. So the desired contact form is $\alpha /(\tilde{H}+\varepsilon)$.

3.5. Hamiltonian $S^{1}$-actions with finitely many fixed points. On the complex projective space $\mathbb{C P}^{n}$, equipped with the Fubini-Study symplectic form for which $\mathbb{C P}^{1} \subset \mathbb{C P}^{n}$ has area $\pi$, the function

$$
H\left(\left[z_{0}: \ldots: z_{n}\right]\right)=\frac{1}{2} \frac{w_{1}\left|z_{1}\right|^{2}+\cdots+w_{n}\left|z_{n}\right|^{2}}{\left|z_{0}\right|^{2}+\cdots+\left|z_{n}\right|^{2}},
$$


where $w_{1}, \ldots, w_{n}$ are integers, is the Hamiltonian for the $S^{1}$-action

$$
\mathrm{e}^{\mathrm{i} \theta}\left[z_{0}: \ldots: z_{n}\right]=\left[z_{0}: \mathrm{e}^{\mathrm{i} w_{1} \theta} z_{1}: \ldots: \mathrm{e}^{\mathrm{i} w_{n} \theta} z_{n}\right] .
$$

When the integers $w_{1}, \ldots, w_{n}$ are pairwise distinct and non-zero, this $S^{1}$-action has the $n+1$ isolated fixed points

$$
[1: 0: \ldots: 0], \ldots,[0: \ldots: 0: 1]
$$

For the action to be effective, we have to assume that the greatest common divisor of $w_{1}, \ldots, w_{n}$ is 1 .

A Hamiltonian $S^{1}$-action extends to the blow-up at any isolated fixed point, see [1, Section IV.3.2], [18, Section 3]. Such a blow-up replaces the one fixed point by $n$ new fixed points. By iterating this procedure, we can realise a Hamiltonian $S^{1}$-action with $n+1+a(n-1)$ fixed points on the blown-up manifold $\mathbb{C P}^{n} \#_{a} \overline{\mathbb{C P}}^{n}$ for any non-negative integer $a$.

Remark 3.10. A blow-up of a symplectic manifold $(B, \omega)$ is effected by removing an open standard ball in a Darboux chart of $(B, \omega)$, and collapsing the characteristic line field on the boundary along which the symplectic form degenerates. This line field defines the Hopf fibration $S^{2 n-1} \rightarrow \mathbb{C P}^{n-1}$, and the collapse produces the exceptional divisor $\mathbb{C P}^{n-1}$. The size of the chosen ball (in terms of the symplectic volume form $\omega^{n}$ ) determines the cohomology class of the symplectic form on the blown-up manifold. By an appropriate choice of the blow-ups we create a symplectic form $\omega_{a}$ on $\mathbb{C P}^{n} \#_{a} \overline{\mathbb{C P}}^{n}$ such that $\left[\omega_{a} / 2 \pi\right]$ is a rational cohomology class, and by rescaling we may assume that this class is integral.

3.6. Laudenbach's surgery construction. Laudenbach 20] describes a surgery construction on hypersurfaces in symplectic manifolds that allows one to control the Hamiltonian flow after the surgery. Inside a single Darboux chart, where the Hamiltonian flow is linear, one performs four simultaneous embedded surgeries of index 1 on the hypersurface. If the hypersurface has dimension $2 n+1$, the belt sphere of an index 1 surgery is $2 n$-dimensional; each surgery replaces two copies of a ball by a cylinder $S^{2 n} \times[-1,1]$. There will be new periodic orbits (i.e. closed characteristics) inside the $(2 n-1)$-dimensional 'equatorial' sphere of that belt sphere. In an explicit model for that surgery, this equatorial sphere can be realised as an irrational ellipsoid, in which case it contains precisely $n$ closed characteristics.

There are Hamiltonian orbits that enter and exit the cylinder $S^{2 n} \times[-1,1]$, and orbits that are trapped inside the cylinder by becoming asymptotic to the equatorial ellipsoid. If one performs only a single surgery, one loses control over the orbits that pass through the cylinder. By performing four such surgeries, arranged in a clever symmetric fashion, Laudenbach ensures that an orbit inside the Darboux chart that enters one of the four cylinders either becomes trapped or does in fact pass through all four of them and exits as if the surgery cylinders had not been there. This is a typical feature of plug constructions, see [14].

Proposition 3.11. On a given $(2 n+1)$-dimensional hypersurface in a symplectic manifold with isolated closed characteristics, one can perform a Laudenbach surgery such that the number of closed characteristics on the new hypersurface has increased by $4 n$ or $4 n-1$. 
Proof. If one performs the Laudenbach construction inside a Darboux chart not traversed by any closed characteristic, one creates a total of $4 n$ additional closed characteristics inside the four surgery cylinders.

One may also choose a Darboux chart traversed by exactly one closed characteristic. In this case one can place the surgery cylinders in such a way that this characteristic becomes trapped inside the first cylinder it enters. So we still get $4 n$ additional closed characteristics, but one of the existing ones has been destroyed.

3.7. Proof of Theorem 3.2. We now put the results of the preceding sections together. First we find a contact form on a Boothby-Wang bundle over $\mathbb{C P}^{n} \#_{a} \overline{\mathbb{C P}}^{n}$ with precisely $n+1+a(n-1)$ periodic Reeb orbits. In dimension five $(n=2)$, this suffices to realise all numbers $k \geq 3=2+1$ as the number of periodic Reeb orbits on some contact manifold. This proves part (a) of the theorem.

In order to prove part (b), we start from an irrational ellipsoid in $\mathbb{R}^{2 n+2}$ with $n+1$ closed characteristics, and we modify this number by performing suitable Laudenbach surgeries on the contact manifold. This allows us to create a connected hypersurface in $\left(\mathbb{R}^{2 n+2}, \omega_{\text {st }}\right)$ with

$$
n+1+b \cdot(4 n-1)+c \cdot 4 n
$$

closed characteristics for any non-negative integers $b, c$.

By choosing $(b, c)$ in the range

$$
(4 n-2,0),(4 n-3,1), \ldots,(1,4 n-3),(0,4 n-2)
$$

we can realise $4 n-1$ successive integers. This implies that starting from

$$
n+1+(4 n-2) \cdot(4 n-1)=16 n^{2}-11 n+3
$$

we can realise all integers. With the help of a Hamiltonian plug [14, one can destroy any isolated closed characteristic in dimension $2 n+1 \geq 5$, so all smaller non-negative integers can likewise be realised.

In dimension $2 n+1=3$, the Hamiltonian plug described in [14] contains two periodic orbits; in other words, one can always destroy an isolated characteristic and create two new ones in the process. This gives us any number $k \geq n+1=2$.

\section{Appendix A. Some quaternionic Calculations}

We write $\langle.,$.$\rangle for the standard inner product on \mathbb{R}^{4}=\mathbb{H}$, and $\operatorname{Re}(\mathbf{a})$ for the real part $a_{0}$ of a quaternion $\mathbf{a}=a_{0}+a_{1} \mathbf{i}+a_{2} \mathbf{j}+a_{3} \mathbf{k}$. The conjugate of $\mathbf{a}$ is $\overline{\mathbf{a}}=a_{0}-a_{1} \mathbf{i}-a_{2} \mathbf{j}-a_{3} \mathbf{k}$.

We identify $S^{3}$ with the unit sphere in $\mathbb{H}$, and $\mathbb{R}^{3}$ with the space of pure imaginary quaternions.

In the following calculations we shall freely use a few basic rules that are easy to verify (or see [10, Section 10.4]). These are:

(i) $\overline{\mathbf{a} \cdot \mathbf{b}}=\overline{\mathbf{b}} \cdot \overline{\mathbf{a}}$;

(ii) $\langle\mathbf{a}, \mathbf{b}\rangle=\operatorname{Re}(\mathbf{a} \cdot \overline{\mathbf{b}})$;

(iii) $\operatorname{Re}(\mathbf{a} \cdot \mathbf{b})=\operatorname{Re}(\mathbf{b} \cdot \mathbf{a})$;

(iv) $\langle\mathbf{x}, \mathbf{y}\rangle=\langle\mathbf{u x} \overline{\mathbf{u}}, \mathbf{u y} \overline{\mathbf{u}}\rangle$ for $\mathbf{x}, \mathbf{y} \in \mathbb{R}^{3}$ and $\mathbf{u} \in S^{3}$.

From (ii) it follows that right-multiplication by a unit quaternion defines an element of $\mathrm{SO}(4)$; with (iii) the same is true for left-multiplication. 
Proof of Lemma 2.11. We first compute $\alpha_{\mathbf{j}}$ in quaternionic notation. This gives

$$
\begin{aligned}
\left(\alpha_{\mathbf{j}}\right)_{\mathbf{u}}(\dot{\mathbf{u}}) & =-2(\mathrm{~d} r \circ \mathbf{j})_{\mathbf{u}}(\dot{\mathbf{u}}) \\
& =-2\langle\mathbf{u}, \mathbf{j} \dot{\mathbf{u}}\rangle \\
& =2 \operatorname{Re}(\dot{\mathbf{u}} \dot{\overline{\mathbf{u}}}) \\
& =2 \operatorname{Re}(\mathbf{j} \mathbf{\overline { u }})
\end{aligned}
$$

For $\alpha_{\mathbf{i}}$ and $\alpha_{\mathbf{k}}$ we have the analogous expression.

The differential of $\Phi$ is given by

$$
T_{\mathbf{u}} \Phi(\dot{\mathbf{u}})=(\dot{\overline{\mathbf{u}}} \mathbf{u} \mathbf{u} \mathbf{\mathbf { u }} \dot{\mathbf{u}}, \dot{\overline{\mathbf{u}}} \mathbf{k u}+\overline{\mathbf{u}} \mathbf{k} \dot{\mathbf{u}}) .
$$

We then compute

$$
\begin{aligned}
& \left(\Phi^{*} \lambda_{1}\right)_{\mathbf{u}}(\dot{\mathbf{u}})=\left(\lambda_{1}\right)_{\Phi(\mathbf{u})}(\dot{\overline{\mathbf{u}} \mathbf{i} \mathbf{u}}+\overline{\mathbf{u}} \mathbf{i} \dot{\mathbf{u}}, \dot{\overline{\mathbf{u}}} \mathbf{k u}+\overline{\mathbf{u}} \mathbf{k} \dot{\mathbf{u}}) \\
& =\langle\overline{\mathbf{u}} \mathbf{k u}, \dot{\overline{\mathbf{u}}} \mathbf{i u}+\overline{\mathbf{u}} \mathbf{i} \dot{\mathbf{u}}\rangle \\
& =\langle\mathbf{k}, \dot{\mathbf{u}} \dot{\mathbf{u}} \mathbf{i}+\mathbf{i} \dot{\mathbf{u}} \overline{\mathbf{u}}\rangle \\
& =\operatorname{Re}(-\mathbf{j} \dot{\mathbf{u}} \overline{\mathbf{u}}-\mathbf{k u} \dot{\bar{u} \mathbf{i}}) \\
& =\operatorname{Re}(-\mathbf{j u} \overline{\mathbf{u}}+\mathbf{j u} \dot{\overline{\mathbf{u}}}) \\
& =2 \operatorname{Re}(\mathbf{j u} \dot{\overline{\mathbf{u}}}),
\end{aligned}
$$

where we have used that the condition $|\mathbf{u}|=1$ gives $\dot{\mathbf{u}} \overline{\mathbf{u}}+\mathbf{u} \dot{\overline{\mathbf{u}}}=0$. So we have shown that $\Phi^{*} \lambda_{1}=\alpha_{\mathbf{j}}$.

For the verification of $\Phi^{*} \lambda_{2}=\alpha_{\mathbf{k}}$ we start from the observation that the standard complex structure of $S^{2}$ acts on $T_{\mathbf{x}} S^{2}$ by $\dot{\mathbf{x}} \mapsto \mathbf{x} \times \dot{\mathbf{x}}$. This gives

$$
\left(\Phi^{*} \lambda_{2}\right)_{\mathbf{u}}(\dot{\mathbf{u}})=\langle\overline{\mathbf{u}} \mathbf{u} \mathbf{u} \times \overline{\mathbf{u}} \mathbf{k u}, \dot{\mathbf{u}} \mathbf{i u}+\overline{\mathbf{u}} \mathbf{u} \dot{\mathbf{u}}\rangle=-\langle\overline{\mathbf{u}} \mathbf{j u}, \dot{\mathbf{u}} \mathbf{i u}+\overline{\mathbf{u}} \mathbf{i} \dot{\mathbf{u}}\rangle .
$$

The further computation is similar.

Acknowledgements. H. G. would like to take this opportunity to thank Jesús Gonzalo for the long-term collaboration on all aspects of contact circles and spheres. The influence of that project on the present work is plainly evident. We also thank Silvia Sabatini for useful conversations and references about Hamiltonian actions, and Janko Latschev for spotting an incomplete argument in an earlier version of this paper.

\section{REFERENCES}

[1] M. Audin, The Topology of Torus Actions on Symplectic Manifolds, Progr. Math. 93 (Birkhäuser Verlag, Basel, 1991).

[2] G. Benedetti, Magnetic Katok examples on the two-sphere, Bull. London Math. Soc. 48 (2016), 855-865.

[3] W. M. Boothby and H. C. Wang, On contact manifolds, Ann. of Math. (2) 68 (1958), $721-734$.

[4] G. E. Bredon, Topology and Geometry, Grad. Texts in Math. 139 (Springer-Verlag, Berlin, 1993)

[5] K. Cieliebak, Symplectic boundaries: creating and destroying closed characteristics, Geom. Funct. Anal. 7 (1997), 269-321.

[6] D. Cristofaro-Gardiner and M. Hutchings, From one Reeb orbit to two, J. Differential Geom. 102 (2016), 25-36.

[7] D. Cristofaro-Gardiner, M. Hutchings and D. Pomerleano, Torsion contact forms in three dimensions have two or infinitely many Reeb orbits, arXiv:1701.02262.

[8] M. Dörner, H. Geiges and K. Zehmisch, Finsler geodesics, periodic Reeb orbits, and open books, arXiv:1611.10035. 
[9] H. Geiges, An Introduction to Contact Topology, Cambridge Stud. Adv. Math. 109 (Cambridge University Press, Cambridge, 2008).

[10] H. Geiges, The Geometry of Celestial Mechanics, London Math. Soc. Stud. Texts 83 (Cambridge University Press, Cambridge, 2016).

[11] H. Geiges and J. Gonzalo, Contact geometry and complex surfaces, Invent. Math. 121 (1995), 147-209.

[12] H. Geiges and J. Gonzalo, Moduli of contact circles, J. Reine Angew. Math. 551 (2002), $41-85$.

[13] H. Geiges and J. Gonzalo PÉrez, Contact spheres and hyperkähler geometry, Comm. Math. Phys. 287 (2009), 719-748.

[14] H. Geiges, N. Röttgen and K. Zehmisch, From a Reeb orbit trap to a Hamiltonian plug, Arch. Math. (Basel) 107 (2016), 397-404.

[15] J. Gonzalo PÉrez, Variedades y Geometría: un curso breve (Ediciones de la Universidad Autónoma de Madrid, 2005).

[16] V. Guillemin, V. Ginzburg and Y. Karshon, Moment Maps, Cobordisms, and Hamiltonian Group Actions, Math. Surveys Monogr. 98 (American Mathematical Society, Providence, RI, 2002).

[17] A. Harris and G. P. Paternain, Dynamically convex Finsler metrics and $J$-holomorphic embeddings of asymptotic cylinders, Ann. Global Anal. Geom. 34 (2008), 115-134.

[18] Y. KARSHON AND L. KESSLER, Circle and torus actions on equal symplectic blow-ups of $\mathbb{C P}^{2}$, Math. Res. Lett. 14 (2007), 807-823.

[19] A. B. Kaток, Ergodic perturbations of degenerate integrable Hamiltonian systems, Math. USSR-Izv. 7 (1973), 535-571.

[20] F. Laudenbach, Trois constructions en topologie symplectique, Ann. Fac. Sci. Toulouse Math. (6) 6 (1997), 697-709.

[21] E. Lerman, On maximal tori in the contactomorphism groups of regular contact manifolds, arXiv: math/0212043.

[22] I. Mundet i Riera, Lifts of smooth group actions to line bundles, Bull. London Math. Soc. 33 (2001), 351-361.

[23] I. M. Singer And J. A. Thorpe, Lecture Notes on Elementary Topology and Geometry (Scott, Foresman and Co., Glenview, Ill., 1967).

[24] C. H. Taubes, The Seiberg-Witten equations and the Weinstein conjecture, Geom. Topol. 11 (2007), 2117-2202.

[25] W. Ziller, Geometry of the Katok examples, Ergodic Theory Dynam. Systems 3 (1982), $135-157$.

Mathematisches Institut, Universität Heidelberg, Im Neuenheimer Feld 205, 69120 HEIDELBERG

E-mail address: palbers@mathi.uni-heidelberg.de

Mathematisches Institut, Universität ZU Köln, Weyertal 86-90, 50931 Köln, GerMANY

E-mail address: geiges@math.uni-koeln.de

Mathematisches Institut, WWU Münster, Einsteinstrasse 62, 48149 Münster, GerMANY

E-mail address: kai.zehmisch@uni-muenster.de 